\title{
Association between the ACYP2 Polymorphisms and IgAN Risk in the Chinese Han Population
}

\author{
Gang Jin ${ }^{a}$ b Yan Liang ${ }^{b} \quad$ Xiaohui Yan ${ }^{b}$ Linping Zhang ${ }^{b}$ Zhenjiang Li ${ }^{b}$ \\ Aiping Yin ${ }^{a}$ Xiaoming Wang ${ }^{b}$ Puxun Tian ${ }^{a}$ \\ aDepartment of Nephrology, The First Affiliated Hospital School of Medicine, Xi'an Jiaotong \\ University, Xi'an, China; ${ }^{b}$ Renal Hemodialysis Center, Shaanxi Provincial People's Hospital, \\ Xi'an, China
}

\section{Keywords}

IgA nephropathy · ACYP2 - Polymorphisms - Case-control study · Chinese Han

\begin{abstract}
Background/Aims: The association between ACYP2 (Acylphosphatase 2) polymorphisms and immunoglobulin A nephropathy (IgAN) risk in the Chinese Han population remains unclear. We aimed to evaluate the association between ACYP2 polymorphisms and IgAN risk by performing a case-control study. Methods: Eleven ACYP2 single nucleotide polymorphisms (SNPs) from $416 \mathrm{lgAN}$ patients and 495 healthy controls were genotyped using the Sequenom MassARRAY platform. Odds ratio (OR) and 95\% confidence interval (Cl) were calculated to evaluate the association of ACYP2 polymorphisms with IgAN risk. Results: We observed that rs843720 was significantly associated with an increased risk of IgAN (allele G: OR $=1.23,95 \%$ Cl: 1.01-1.49, $p=0.036$; dominant model: $\mathrm{OR}=1.55,95 \% \mathrm{Cl}: 1.01-2.37, p=0.044$; log-additive model: $\mathrm{OR}=1.43,95 \% \mathrm{Cl}: 1.04-1.95, p=0.026)$ before Bonferroni correction. The SNP rs12615793 was also significantly associated with an increased IgAN risk in the recessive model $(\mathrm{OR}=3.33,95 \% \mathrm{Cl}: 1.05-10.51, p=0.042)$ before Bonferroni correction. Conclusion: These findings suggested that polymorphisms (rs843720 and rs12615793) of ACYP2 may be pivotal in the development of IgAN. However, more functional and association studies with larger sample sizes should be performed to further validate our results in the future.
\end{abstract}




\section{Kidney \\ Blood Pressure \\ Research}

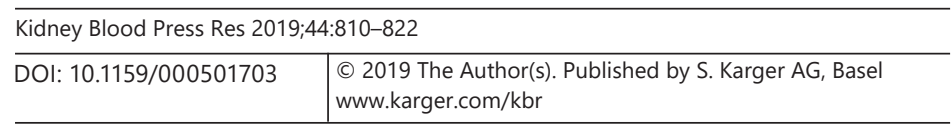

Jin et al.: ACYP2 Polymorphisms and IgAN Risk

\section{Introduction}

The immunoglobulin A nephropathy (IgAN) is the most common form of primary glomerulonephritis in the world and is characterized by the excessive deposition of IgAN in the mesangial area of glomeruli leading to mesangial proliferation and chronic glomerular inflammation $[1,2]$. Previous studies have shown that about $30 \%$ of IgAN patients will progress to end-stage renal disease within 20-25 years of disease onset [3]. Poor prognostic factors for progression of renal damage in patients with IgAN include gender, older age, hypertension, severe proteinuria, renal lesions, and advanced histological involvement [4]. Furthermore, a family history of chronic nephritis, susceptibility to the common cold, preference for salty foods, frequent consumption of raw eggs, and a high intake of carbohydrates are significantly associated with increased IgAN risk [5]. To date, the exact pathogenesis of IgAN remains unclear. However, increasing lines of evidences have suggested the involvement of genetic factors in the pathogenesis of IgAN and variation in clinical manifestation. Evidence derived from experimental models of spontaneous IgAN and studies of the genetics, familial clustering, ethnic and geographic differences in prevalence support the influence of genetic factors in the development and progression of IgAN [6-9]. Previous studies have identified multiple susceptibility polymorphisms for the risk of IgAN [10-13]. Therefore, identifying genes associated with susceptibility to IgAN is particularly important and might provide a genetic target for therapeutic intervention in IgAN.

ACYP2 (Acylphosphatase 2) encodes a small cytosolic enzyme acylphosphatase (11 kDa) that can hydrolyze the phosphoenzyme intermediate of different membrane pumps, particularly the $\mathrm{Ca}^{2+} / \mathrm{Mg}^{2+}$-ATPase from sarcoplasmic reticulum of skeletal muscle [14]. These enzymes can adjust the stability of the inner environment through calcium ion transport [15]. Polak-Jonkisz et al. [16] found that the $\mathrm{Ca}^{2+} / \mathrm{Mg}^{2+}$-ATPase activity is reduced, and $\mathrm{Ca}^{2+}$ homeostasis is disturbed in children with chronic kidney disease. Moreover, it also exacerbates renal function deterioration. In addition, a genome-wide association study found that rs11125529 in $A C Y P 2$ is significantly associated with telomere length [17]. Accumulating evidence prompts the view that telomere length is an indicator for organismal aging. To our best knowledge, aging is one of the contributing risk factors for kidney diseases. It was reported that telomere shortening is associated with human IgAN $[18,19]$. Some association studies have demonstrated that polymorphisms in $A C Y P 2$ are associated with the susceptibility to many diseases, such as high-altitude pulmonary edema [20], breast cancer [21], lung cancer [22], gastric cancer [23], and ischemic stroke [24].

However, to our knowledge, there are no reported studies of the association between ACYP2 polymorphisms and the risk of IgAN among the Chinese Han population. We hypothesized that polymorphisms in ACYP2 are associated with the risk of IgAN. To test this hypothesis, therefore, we conducted a case-control association study to evaluate the association between the ACYP2 polymorphisms and IgAN risk in the Chinese Han population.

\section{Materials and Methods}

\section{Subjects}

In this study, 416 patients diagnosed with IgAN by renal biopsy were enrolled from the First Affiliated Hospital of Xi'an Jiaotong University. 495 unrelated healthy controls were randomly selected from those who went to the aforementioned hospital for medical examination during the same period. Renal biopsy pathological diagnostic criteria established by Zou (2011) were used as diagnostic criteria for IgAN. All the selected individuals are ethnic Han Chinese with no genetic relationship and their ancestors had lived in the region for at 


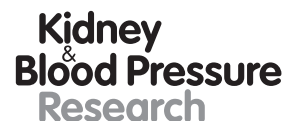

\begin{tabular}{l|l}
\hline Kidney Blood Press Res 2019;44:810-822 \\
\hline DOI: 10.1159/000501703 & $\begin{array}{l}\text { (c) 2019 The Author(s). Published by S. Karger AG, Basel } \\
\text { www.karger.com/kbr }\end{array}$ \\
\hline
\end{tabular}

Jin et al.: ACYP2 Polymorphisms and IgAN Risk

Table 1. The primer sequences for genotyping SNPs in ACYP2

\begin{tabular}{llll}
\hline SNP-ID & 2nd-PCRP & 1st-PCRP & UEP-SEQ \\
\hline rs6713088 & ACGTTGGATGGTCACCAAAACACGTAATG & ACGTTGGATGACACACACAGACTCCTTCAC & gaggcCAGAATGGTCCACTAGAGA \\
rs12621038 & ACGTTGGATGGGCATAAGTTTATTGCCTC & ACGTTGGATGATTGTGCTAGGCACTTTAGG & ccATTGCCTCAGCTAGACT \\
rs1682111 & ACGTTGGATGGCCAGTGGGAATGCAAAATG & ACGTTGGATGGAATTGCTGGGTTATTGGC & tgtcATGCAAAATGAAACAGACACTT \\
rs843752 & ACGTTGGATGGAGACAACATAATGGAGGTC & ACGTTGGATGTCCTCTTTTCAGAAACCTGC & cGAGTTTGGGTTGAGGT \\
rs843645 & ACGTTGGATGACAGTGCCTTTAGCAAGGTG & ACGTTGGATGGAAATCTGAATACCACCTAC & TCATAGGCACTACTGTATC \\
rs11125529 & ACGTTGGATGCGGAAGAAAAGAAGATGAC & ACGTTGGATGGAGCTTAGTTGTTTACAGATG & AGAAAAGAAGATGACTAAAACAT \\
rs12615793 & ACGTTGGATGATCTTGGCCCTTGAAGAA & ACGTTGGATGTTTGAGCTTAGTTGTTTAC & AAATTGAGTGACAAATATAAACTAC \\
rs843711 & ACGTTGGATGTGCCTTGTGGGATTAGAGC & ACGTTGGATGGACAAAGGACCTTACAACTC & gggaTCAGGGAACCAGTGCAAA \\
rs1189664 & ACGTTGGATGTGTCTCTGACCTAGCATGTA & ACGTTGGATGAAGTCAGAATAGTGCTTAC & GTTAAGCTTGCAAGGAG \\
rs17045754 & ACGTTGGATGGAAATCAGGGATATTAGTGC & ACGTTGGATGCTGTAAAAGTTCTGGCATGG & caggTATTCAGCTTCCTAGAGTTA \\
rs843720 & ACGTTGGATGAGTCAGAGCTAGACCTCTGG & ACGTTGGATGCTTCACAACACTCCTGTAAG & cccCAATCTGTCTCAGGGTCTT \\
\hline
\end{tabular}

SNP, single nucleotide polymorphism; PCRP, polymerase chain reaction primer; UEP, unique base extension primer. Sequences are written in the $5^{\prime} \rightarrow 3^{\prime}($ left to right) orientation.

least three generations. Patients with Henoch-Schönlein purpura, systemic lupus erythematosus, allergic purpura, chronic liver diseases, ankylosing spondylitis renal damage, and other secondary IgAN were excluded through detailed clinical and laboratory examinations. The patients were recruited without age, sex, or disease stage restriction.

\section{DNA Extraction}

A 5-mL sample of peripheral venous blood was collected in EDTA vacutainer tube from each participant on an empty stomach in the morning and then placed at $-80^{\circ} \mathrm{C}$ for cryopreservation. Genomic DNA was isolated from whole blood using the GoldMag DNA Purifcation Kit (GoldMag Co. Ltd., Xi'an, China) according to the manufacturer's protocol and preserved at $-20^{\circ} \mathrm{C}$ [25]. The extracted DNA was assessed for concentration and purity utilizing an ultraviolet spectrophotometer (NanoDrop 2000; Thermo Fisher Scientific, Waltham, MA, USA $]$ [26]. The purity was monitored by the $\left(\mathrm{OD}_{260}-\mathrm{OD}_{320}\right) /\left(\mathrm{OD}_{280}-\mathrm{OD}_{320}\right)$ ratio. The DNA concentration was diluted to $10 \mathrm{ng} / \mu \mathrm{L}$ for polymerase chain reaction amplification.

\section{Single Nucleotide Polymorphism Selection and Genotyping}

Eleven single nucleotide polymorphisms (SNPs; rs6713088, rs12621038, rs1682111, rs843752, rs843645, rs11125529, rs12615793, rs843711, rs11896604, rs17045754, and rs843720) in the ACYP2 gene with minor allele frequency $>0.05$ in the Chinese Han Beijing population from the HapMap database and previously reported [20-24] were selected for analysis in our study. The primers of SNPs were designed by the Sequenom MassARRAY Assay Design 3.0 Software (Sequenom, San Diego, CA, USA). The sequences of primers corresponding to each SNP are shown in Table 1. Genotyping of the 11 SNPs were conducted using the Sequenom MassARRAY platform (Sequenom) according to the standard protocol recommended by the manufacturer [27]. Sequenom Typer 4.0 software was used for data management and analysis.

\section{Statistical Analysis}

Whether the genotype frequency distribution of control was consistent with HardyWeinberg equilibrium was estimated using the $\chi^{2}$ test. The difference in the distribution of allele frequencies between cases and controls were compared by the $\chi^{2}$ test, and the odds ratio (OR) and 95\% confidence interval (CI) indicated relative risk. Genetic model and haplotype analyses were applied using unconditional multivariate logistic regression analysis 


\section{Kidney \\ Blood Pressure \\ Research}

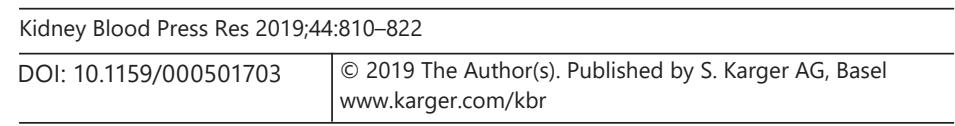

Jin et al.: ACYP2 Polymorphisms and IgAN Risk

Table 2. Basic characteristic of cases and controls in this study

\begin{tabular}{lccc}
\hline Variables & Cases & Controls & $p$ value \\
\hline Total & 416 & 495 & \\
\hline Gender & & & $<0.001$ \\
$\quad$ Female & $145(34.9)$ & $315(63.6)$ & \\
$\quad$ Male & $271(65.1)$ & $180(36.4)$ & \\
\hline Age, years & $33.35 \pm 12.13$ & $54.48 \pm 9.44$ & $<0.001$ \\
\hline
\end{tabular}

Data are presented as $n(\%)$ or mean \pm SD. $p$ values were calculated using Pearson's $\chi^{2}$ test for gender and Student's $t$ test for age. $p<0.05$ indicates statistical significance.

to evaluate the correlation between polymorphisms and IgAN risk. Moreover, we performed Bonferroni correction to eliminate the probability of false-positive findings in the genetic model analyses. Pairwise linkage disequilibrium (LD) between each SNP, quantified as $\mathrm{D}^{\prime}$ and $r^{2}$, was measured by the Haploview software version 4.2 (Broad Institute, Cambridge, MA, USA). The Haploview software was used to test the association between statistically inferred haplotypes and IgAN risk. All statistical analyses were performed with Microsoft Excel (Microsoft Corp., Redmond, WA, USA) and Statistical Package for the Social Sciences (SPSS) version 20.0 (SPSS Inc., Chicago, IL, USA). $p$ value $<0.05$ was considered to indicate that a difference was statistically significant, and all statistical tests were two-sided at a test level of $\alpha=0.05$. Besides, the $p$ value $<0.004(0.05 / 11)$ indicates statistical significance after Bonferroni correction.

\section{Results}

\section{Characteristics of Subjects}

The characteristics of cases and controls enrolled in this study are shown Table 2. This study recruited 911 subjects, including 416 IgAN patients (145 females and 271 males) and 495 healthy controls ( 315 females and 180 males). There was a significant difference between the case and control groups with respect to gender distribution $(p<0.05)$. The average age of IgAN patients was $33.35 \pm 12.13$ years compared with $54.48 \pm 9.44$ years in controls, which also showed statistically significant difference $(p<0.05)$. In order to eliminate those residual confounding effects, the variables of age and gender were adjusted in later multivariate unconditional logistic regression analysis.

\section{Allele Distributions of ACYP2 Polymorphisms}

The allele distribution of the selected SNPs in cases and controls is shown in Table 3. The genotypic distribution of all SNPs in the control group conformed to Hardy-Weinberg equilibrium $(p>0.05)$. We found that the frequency of rs843720 G allele in cases (38.5\%) was higher than in the controls (33.7\%), and the $\mathrm{G}$ allele of rs843720 was significantly associated with an increased the risk of IgAN (OR = 1.23, 95\% CI: 1.01-1.49, $p=0.036)$. However, there was no significant difference in other allele distributions of $A C Y P 2$ polymorphisms between IgAN patients and controls.

\section{Genetic Model Analyses}

Genetic model analysis indicated that the AA genotype of rs12615793 was significantly associated with an increased risk of IgAN, compared to the GG and AG genotypes (adjusted 
Table 3. Allele distribution of ACYP2 polymorphisms and association with IgAN risk

\begin{tabular}{|c|c|c|c|c|c|c|c|c|c|c|}
\hline SNP-ID & Position & Band & $\begin{array}{l}\text { Alleles } \\
\text { A/B }\end{array}$ & Role & HWE- $p$ & $\begin{array}{l}\text { Case } \\
\text { (MAF) }\end{array}$ & $\begin{array}{l}\text { Control } \\
\text { (MAF) }\end{array}$ & ORs & $95 \% \mathrm{CI}$ & $p$ \\
\hline rs6713088 & 54345469 & $2 \mathrm{p} 16.2$ & $\mathrm{G} / \mathrm{C}$ & Intron & 0.778 & 0.409 & 0.397 & 1.05 & $0.87-1.27$ & 0.606 \\
\hline rs12621038 & 54391113 & $2 \mathrm{p} 16.2$ & $\mathrm{~T} / \mathrm{C}$ & Intron & 0.786 & 0.449 & 0.457 & 0.97 & $0.80-1.16$ & 0.715 \\
\hline rs1682111 & 54427979 & $2 \mathrm{p} 16.2$ & $\mathrm{~A} / \mathrm{T}$ & Intron & 0.832 & 0.323 & 0.303 & 1.10 & $0.90-1.34$ & 0.362 \\
\hline rs843752 & 54446587 & $2 \mathrm{p} 16.2$ & $\mathrm{G} / \mathrm{T}$ & Intron & 0.569 & 0.263 & 0.269 & 0.97 & $0.79-1.20$ & 0.793 \\
\hline rs843645 & 54474664 & $2 \mathrm{p} 16.2$ & $\mathrm{G} / \mathrm{T}$ & Downstream & 1.000 & 0.246 & 0.261 & 0.93 & $0.75-1.15$ & 0.488 \\
\hline rs11125529 & 54475866 & $2 \mathrm{p} 16.2$ & $\mathrm{~A} / \mathrm{C}$ & Downstream & 0.092 & 0.195 & 0.180 & 1.10 & $0.87-1.40$ & 0.416 \\
\hline rs12615793 & 54475914 & $2 \mathrm{p} 16.2$ & $\mathrm{~A} / \mathrm{G}$ & Downstream & 0.242 & 0.212 & 0.191 & 1.13 & $0.90-1.43$ & 0.283 \\
\hline rs843711 & 54479117 & $2 \mathrm{p} 16.2$ & $\mathrm{C} / \mathrm{T}$ & Downstream & 0.124 & 0.460 & 0.454 & 1.03 & $0.85-1.24$ & 0.778 \\
\hline rs11896604 & 54479199 & $2 \mathrm{p} 16.2$ & $\mathrm{G} / \mathrm{C}$ & Downstream & 0.249 & 0.214 & 0.194 & 1.13 & $0.90-1.42$ & 0.290 \\
\hline rs17045754 & 54496757 & $2 \mathrm{p} 16.2$ & $\mathrm{C} / \mathrm{G}$ & Intron & 0.297 & 0.197 & 0.185 & 1.08 & $0.86-1.37$ & 0.507 \\
\hline rs843720 & 54510660 & $2 \mathrm{p} 16.2$ & $\mathrm{G} / \mathrm{T}$ & Intron & 0.422 & 0.385 & 0.337 & 1.23 & $1.01-1.49$ & 0.036 \\
\hline
\end{tabular}

SNP, single nucleotide polymorphism; A, minor alleles; B, major alleles; HWE, Hardy-Weinberg equilibrium; MAF, minor allele frequency; OR, odds ratio; 95\% CI: $95 \%$ confidence interval. $p<0.05$ indicates statistical significance. $p$ value $<0.004(0.05 / 11)$ indicates statistical significance after Bonferroni correction.

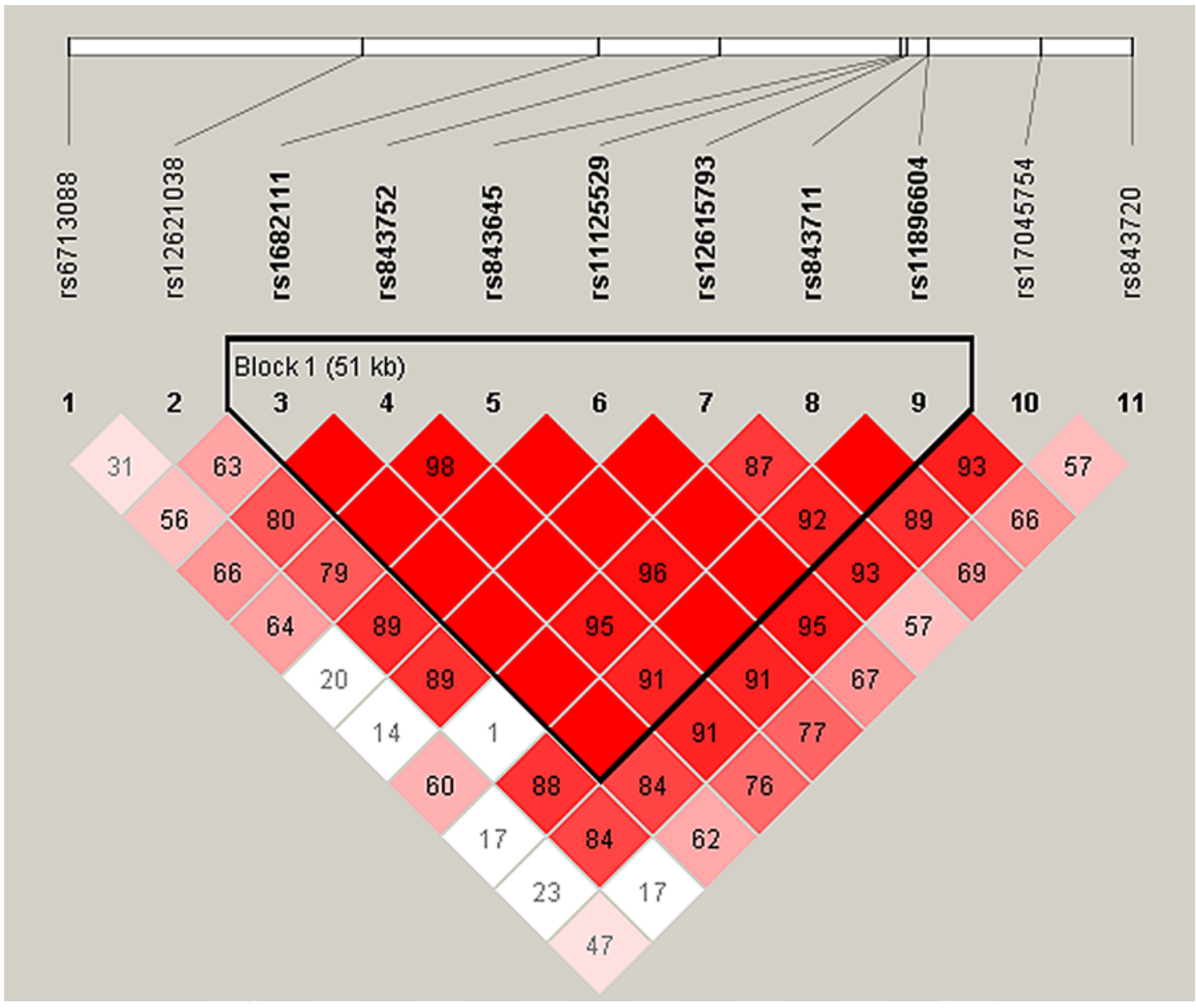

Fig. 1. Haplotype block map for the eleven SNPs in ACYP2. The LD between each pair of SNPs is standardized $\mathrm{D}^{\prime}$, bright red corresponding to very strong $\mathrm{LD}$, white corresponding to no $\mathrm{LD}$, and pink corresponding to intermediate LD. 
Table 4. Genetic model analyses of the association between ACYP2 polymorphisms and IgAN risk

\begin{tabular}{|c|c|c|c|c|c|c|c|c|}
\hline \multirow[t]{2}{*}{ SNP-ID } & \multirow[t]{2}{*}{ Model } & \multirow[t]{2}{*}{ Genotype } & \multirow[t]{2}{*}{ Case, \% } & \multirow[t]{2}{*}{ Control, \% } & \multicolumn{2}{|c|}{ Without adjustment } & \multicolumn{2}{|l|}{ With adjustment } \\
\hline & & & & & OR (95\% CI) & $p$ & OR $(95 \%$ CI) & $p$ \\
\hline \multirow[t]{10}{*}{ rs6713088 } & Codominant & $\mathrm{C} / \mathrm{C}$ & 143 (34.4\%) & $178(36 \%)$ & 1.00 & 0.860 & 1.00 & 0.580 \\
\hline & & $\mathrm{C} / \mathrm{G}$ & $206(49.5 \%)$ & $240(48.6 \%)$ & $1.07(0.80-1.43)$ & & $0.95(0.60-1.50)$ & \\
\hline & & $\mathrm{G} / \mathrm{G}$ & 67 (16.1\%) & $76(15.4 \%)$ & $1.10(0.74-1.63)$ & & $0.72(0.39-1.35)$ & \\
\hline & Dominant & $\mathrm{C} / \mathrm{C}$ & $143(34.4 \%)$ & $178(36 \%)$ & 1.00 & 0.600 & 1.00 & 0.590 \\
\hline & & $\mathrm{C} / \mathrm{G}-\mathrm{G} / \mathrm{G}$ & $273(65.6 \%)$ & $316(64 \%)$ & $1.08(0.82-1.41)$ & & $0.89(0.58-1.37)$ & \\
\hline & Recessive & $\mathrm{C} / \mathrm{C}-\mathrm{C} / \mathrm{G}$ & 349 (83.9\%) & $418(84.6 \%)$ & 1.00 & 0.770 & 1.00 & 0.310 \\
\hline & & $\mathrm{G} / \mathrm{G}$ & 67 (16.1\%) & $76(15.4 \%)$ & $1.06(0.74-1.51)$ & & $0.75(0.43-1.31)$ & \\
\hline & Overdominant & $\mathrm{C} / \mathrm{C}-\mathrm{G} / \mathrm{G}$ & $210(50.5 \%)$ & $254(51.4 \%)$ & 1.00 & 0.780 & 1.00 & 0.810 \\
\hline & & $\mathrm{C} / \mathrm{G}$ & $206(49.5 \%)$ & $240(48.6 \%)$ & $1.04(0.80-1.35)$ & & $1.05(0.70-1.59)$ & \\
\hline & Log-additive & - & - & - & $1.05(0.87-1.27)$ & 0.600 & $0.87(0.64-1.17)$ & 0.360 \\
\hline \multirow[t]{10}{*}{ rs12621038 } & Codominant & $\mathrm{C} / \mathrm{C}$ & $120(29.2 \%)$ & 147 (29.8\%) & 1.00 & 0.620 & 1.00 & 0.850 \\
\hline & & $\mathrm{T} / \mathrm{C}$ & 213 (51.8\%) & $242(49 \%)$ & $1.08(0.80-1.46)$ & & $0.88(0.55-1.42)$ & \\
\hline & & $\mathrm{T} / \mathrm{T}$ & 78 (19\%) & $105(21.3 \%)$ & $0.91(0.62-1.33)$ & & $0.99(0.55-1.80)$ & \\
\hline & Dominant & $\mathrm{C} / \mathrm{C}$ & $120(29.2 \%)$ & 147 (29.8\%) & 1.00 & 0.850 & 1.00 & 0.690 \\
\hline & & $\mathrm{T} / \mathrm{C}-\mathrm{T} / \mathrm{T}$ & $291(70.8 \%)$ & 347 (70.2\%) & 1.03 (0.77-1.37) & & $0.91(0.58-1.43)$ & \\
\hline & Recessive & $\mathrm{C} / \mathrm{C}-\mathrm{T} / \mathrm{C}$ & $333(81 \%)$ & $389(78.7 \%)$ & 1.00 & 0.400 & 1.00 & 0.790 \\
\hline & & $\mathrm{T} / \mathrm{T}$ & 78 (19\%) & $105(21.3 \%)$ & $0.87(0.63-1.20)$ & & $1.07(0.64-1.80)$ & \\
\hline & Overdominant & $\mathrm{C} / \mathrm{C}-\mathrm{T} / \mathrm{T}$ & $198(48.2 \%)$ & $252(51 \%)$ & 1.00 & 0.400 & 1.00 & 0.560 \\
\hline & & $\mathrm{T} / \mathrm{C}$ & $213(51.8 \%)$ & $242(49 \%)$ & $1.12(0.86-1.46)$ & & $0.88(0.58-1.34)$ & \\
\hline & Log-additive & - & - & - & $0.97(0.80-1.16)$ & 0.710 & $0.98(0.73-1.32)$ & 0.910 \\
\hline \multirow[t]{10}{*}{ rs1682111 } & Codominant & $\mathrm{T} / \mathrm{T}$ & $185(44.6 \%)$ & $239(48.3 \%)$ & 1.00 & 0.530 & 1.00 & 0.510 \\
\hline & & $\mathrm{T} / \mathrm{A}$ & $192(46.3 \%)$ & $212(42.8 \%)$ & $1.17(0.89-1.54)$ & & $1.15(0.74-1.78)$ & \\
\hline & & $\mathrm{A} / \mathrm{A}$ & 38 (9.2\%) & 44 (8.9\%) & $1.12(0.69-1.79)$ & & $1.52(0.73-3.17)$ & \\
\hline & Dominant & $\mathrm{T} / \mathrm{T}$ & $185(44.6 \%)$ & $239(48.3 \%)$ & 1.00 & 0.260 & 1.00 & 0.380 \\
\hline & & $\mathrm{T} / \mathrm{A}-\mathrm{A} / \mathrm{A}$ & $230(55.4 \%)$ & $256(51.7 \%)$ & $1.16(0.89-1.51)$ & & $1.20(0.79-1.83)$ & \\
\hline & Recessive & $\mathrm{T} / \mathrm{T}-\mathrm{T} / \mathrm{A}$ & 377 (90.8\%) & $451(91.1 \%)$ & 1.00 & 0.890 & 1.00 & 0.330 \\
\hline & & $\mathrm{A} / \mathrm{A}$ & 38 (9.2\%) & 44 (8.9\%) & $1.03(0.66-1.63)$ & & $1.42(0.70-2.86)$ & \\
\hline & Overdominant & $\mathrm{T} / \mathrm{T}-\mathrm{A} / \mathrm{A}$ & $223(53.7 \%)$ & $283(57.2 \%)$ & 1.00 & 0.300 & 1.00 & 0.760 \\
\hline & & $\mathrm{T} / \mathrm{A}$ & $192(46.3 \%)$ & $212(42.8 \%)$ & $1.15(0.88-1.49)$ & & $1.07(0.70-1.62)$ & \\
\hline & Log-additive & - & - & - & $1.10(0.90-1.35)$ & 0.350 & $1.20(0.87-1.65)$ & 0.270 \\
\hline \multirow[t]{10}{*}{ rs843752 } & Codominant & $\mathrm{T} / \mathrm{T}$ & $228(54.8 \%)$ & $262(52.9 \%)$ & 1.00 & 0.690 & 1.00 & 0.630 \\
\hline & & $\mathrm{G} / \mathrm{T}$ & 157 (37.7\%) & $200(40.4 \%)$ & $0.90(0.69-1.19)$ & & $0.81(0.52-1.25)$ & \\
\hline & & $\mathrm{G} / \mathrm{G}$ & $31(7.5 \%)$ & $33(6.7 \%)$ & $1.08(0.64-1.82)$ & & $0.91(0.39-2.10)$ & \\
\hline & Dominant & $\mathrm{T} / \mathrm{T}$ & 228 (54.8\%) & $262(52.9 \%)$ & 1.00 & 0.570 & 1.00 & 0.350 \\
\hline & & $\mathrm{G} / \mathrm{T}-\mathrm{G} / \mathrm{G}$ & $188(45.2 \%)$ & 233 (47.1\%) & $0.93(0.71-1.20)$ & & $0.82(0.54-1.24)$ & \\
\hline & Recessive & $\mathrm{T} / \mathrm{T}-\mathrm{G} / \mathrm{T}$ & 385 (92.5\%) & $462(93.3 \%)$ & 1.00 & 0.640 & 1.00 & 0.990 \\
\hline & & $\mathrm{G} / \mathrm{G}$ & $31(7.5 \%)$ & $33(6.7 \%)$ & $1.13(0.68-1.87)$ & & $1.00(0.44-2.25)$ & \\
\hline & Overdominant & $\mathrm{T} / \mathrm{T}-\mathrm{G} / \mathrm{G}$ & $259(62.3 \%)$ & 295 (59.6\%) & 1.00 & 0.410 & 1.00 & 0.350 \\
\hline & & $\mathrm{G} / \mathrm{T}$ & 157 (37.7\%) & $200(40.4 \%)$ & $0.89(0.68-1.17)$ & & $0.82(0.54-1.25)$ & \\
\hline & Log-additive & - & - & - & $0.97(0.79-1.20)$ & 0.790 & $0.88(0.63-1.23)$ & 0.450 \\
\hline
\end{tabular}


Table 4 (continued)

\begin{tabular}{|c|c|c|c|c|c|c|c|c|}
\hline \multirow[t]{2}{*}{ SNP-ID } & \multirow[t]{2}{*}{ Model } & \multirow[t]{2}{*}{ Genotype } & \multirow[t]{2}{*}{ Case, $\%$} & \multirow[t]{2}{*}{ Control, \% } & \multicolumn{2}{|c|}{ Without adjustment } & \multicolumn{2}{|l|}{ With adjustment } \\
\hline & & & & & OR $(95 \% \mathrm{CI})$ & $p$ & OR $(95 \%$ CI $)$ & $p$ \\
\hline \multirow[t]{10}{*}{ rs843645 } & Codominant & $\mathrm{T} / \mathrm{T}$ & $236(56.7 \%)$ & $270(54.5 \%)$ & 1.00 & 0.780 & 1.00 & 0.550 \\
\hline & & $\mathrm{G} / \mathrm{T}$ & $155(37.3 \%)$ & $192(38.8 \%)$ & $0.92(0.70-1.22)$ & & $0.84(0.54-1.30)$ & \\
\hline & & $\mathrm{G} / \mathrm{G}$ & $25(6 \%)$ & 33 (6.7\%) & $0.87(0.50-1.50)$ & & $0.67(0.28-1.61)$ & \\
\hline & Dominant & $\mathrm{T} / \mathrm{T}$ & $236(56.7 \%)$ & $270(54.5 \%)$ & 1.00 & 0.510 & 1.00 & 0.330 \\
\hline & & $\mathrm{G} / \mathrm{T}-\mathrm{G} / \mathrm{G}$ & $180(43.3 \%)$ & $225(45.5 \%)$ & $0.92(0.70-1.19)$ & & $0.81(0.54-1.23)$ & \\
\hline & Recessive & $\mathrm{T} / \mathrm{T}-\mathrm{G} / \mathrm{T}$ & $391(94 \%)$ & $462(93.3 \%)$ & 1.00 & 0.690 & 1.00 & 0.450 \\
\hline & & $\mathrm{G} / \mathrm{G}$ & $25(6 \%)$ & 33 (6.7\%) & $0.90(0.52-1.53)$ & & $0.72(0.31-1.70)$ & \\
\hline & Overdominant & $\mathrm{T} / \mathrm{T}-\mathrm{G} / \mathrm{G}$ & $261(62.7 \%)$ & $303(61.2 \%)$ & 1.00 & 0.640 & 1.00 & 0.540 \\
\hline & & $\mathrm{G} / \mathrm{T}$ & $155(37.3 \%)$ & $192(38.8 \%)$ & $0.94(0.72-1.23)$ & & $0.87(0.57-1.34)$ & \\
\hline & Log-additive & - & - & - & $0.93(0.75-1.15)$ & 0.490 & $0.83(0.59-1.16)$ & 0.280 \\
\hline \multirow[t]{10}{*}{ rs11125529 } & Codominant & $\mathrm{C} / \mathrm{C}$ & $271(65.1 \%)$ & 327 (66.1\%) & 1.00 & 0.180 & 1.00 & 0.290 \\
\hline & & $\mathrm{C} / \mathrm{A}$ & $128(30.8 \%)$ & $158(31.9 \%)$ & $0.98(0.74-1.30)$ & & $0.85(0.54-1.33)$ & \\
\hline & & $\mathrm{A} / \mathrm{A}$ & 17 (4.1\%) & $10(2 \%)$ & $2.05(0.92-4.55)$ & & $2.40(0.66-8.81)$ & \\
\hline & Dominant & $\mathrm{C} / \mathrm{C}$ & $271(65.1 \%)$ & $327(66.1 \%)$ & 1.00 & 0.770 & 1.00 & 0.700 \\
\hline & & $\mathrm{C} / \mathrm{A}-\mathrm{A} / \mathrm{A}$ & 145 (34.9\%) & $168(33.9 \%)$ & $1.04(0.79-1.37)$ & & $0.92(0.59-1.42)$ & \\
\hline & Recessive & $\mathrm{C} / \mathrm{C}-\mathrm{C} / \mathrm{A}$ & 399 (95.9\%) & $485(98 \%)$ & 1.00 & 0.067 & 1.00 & 0.160 \\
\hline & & $\mathrm{A} / \mathrm{A}$ & $17(4.1 \%)$ & $10(2 \%)$ & $2.07(0.94-4.56)$ & & $2.53(0.70-9.19)$ & \\
\hline & Overdominant & $\mathrm{C} / \mathrm{C}-\mathrm{A} / \mathrm{A}$ & $288(69.2 \%)$ & $337(68.1 \%)$ & 1.00 & 0.710 & 1.00 & 0.380 \\
\hline & & $\mathrm{C} / \mathrm{A}$ & $128(30.8 \%)$ & $158(31.9 \%)$ & $0.95(0.72-1.26)$ & & $0.82(0.52-1.28)$ & \\
\hline & Log-additive & - & - & - & $1.11(0.87-1.41)$ & 0.410 & $1.01(0.69-1.49)$ & 0.950 \\
\hline \multirow[t]{10}{*}{ rs12615793 } & Codominant & $\mathrm{G} / \mathrm{G}$ & $260(62.5)$ & $316(64)$ & 1.00 & 0.100 & 1.00 & 0.061 \\
\hline & & $A / G$ & $136(32.7)$ & 167 (33.8) & $0.99(0.75-1.31)$ & & $0.76(0.48-1.19)$ & \\
\hline & & $\mathrm{A} / \mathrm{A}$ & $20(4.8)$ & $11(2.2)$ & $2.21(1.04-4.70)$ & & $3.04(0.95-9.72)$ & \\
\hline & Dominant & $\mathrm{G} / \mathrm{G}$ & $260(62.5)$ & $316(64)$ & 1.00 & 0.650 & 1.00 & 0.500 \\
\hline & & $\mathrm{A} / \mathrm{G}-\mathrm{A} / \mathrm{A}$ & $156(37.5)$ & $178(36)$ & $1.07(0.81-1.40)$ & & $0.86(0.56-1.33)$ & \\
\hline & Recessive & $\mathrm{G} / \mathrm{G}-\mathrm{A} / \mathrm{G}$ & 396 (95.2) & 483 (97.8) & 1.00 & 0.032 & 1.00 & 0.042 \\
\hline & & $\mathrm{A} / \mathrm{A}$ & $20(4.8)$ & $11(2.2)$ & $2.22(1.05-4.68)$ & & $3.33(1.05-10.51)$ & \\
\hline & Overdominant & $\mathrm{G} / \mathrm{G}-\mathrm{A} / \mathrm{A}$ & $280(67.3)$ & 327 (66.2) & 1.00 & 0.720 & 1.00 & 0.150 \\
\hline & & $\mathrm{A} / \mathrm{G}$ & $136(32.7)$ & 167 (33.8) & $0.95(0.72-1.25)$ & & $0.72(0.46-1.12)$ & \\
\hline & Log-additive & - & - & - & $1.14(0.90-1.44)$ & 0.270 & $1.01(0.69-1.47)$ & 0.960 \\
\hline \multirow[t]{10}{*}{ rs843711 } & Codominant & $\mathrm{C} / \mathrm{C}$ & $125(30.2 \%)$ & $139(28.1 \%)$ & 1.00 & 0.220 & 1.00 & 0.480 \\
\hline & & $\mathrm{C} / \mathrm{T}$ & $197(47.6 \%)$ & $263(53.1 \%)$ & $0.83(0.61-1.13)$ & & $0.77(0.48-1.25)$ & \\
\hline & & $\mathrm{T} / \mathrm{T}$ & $92(22.2 \%)$ & 93 (18.8\%) & $1.10(0.76-1.60)$ & & $0.72(0.39-1.32)$ & \\
\hline & Dominant & $\mathrm{C} / \mathrm{C}$ & $125(30.2 \%)$ & $139(28.1 \%)$ & 1.00 & 0.480 & 1.00 & 0.240 \\
\hline & & $\mathrm{C} / \mathrm{T}-\mathrm{T} / \mathrm{T}$ & $289(69.8 \%)$ & $356(71.9 \%)$ & $0.90(0.68-1.20)$ & & $0.76(0.48-1.20)$ & \\
\hline & Recessive & $\mathrm{C} / \mathrm{C}-\mathrm{C} / \mathrm{T}$ & $322(77.8 \%)$ & $402(81.2 \%)$ & 1.00 & 0.200 & 1.00 & 0.540 \\
\hline & & $\mathrm{T} / \mathrm{T}$ & $92(22.2 \%)$ & 93 (18.8\%) & $1.24(0.89-1.71)$ & & $0.85(0.51-1.43)$ & \\
\hline & Overdominant & $\mathrm{C} / \mathrm{C}-\mathrm{T} / \mathrm{T}$ & 217 (52.4\%) & $232(46.9 \%)$ & 1.00 & 0.096 & 1.00 & 0.560 \\
\hline & & $\mathrm{C} / \mathrm{T}$ & $197(47.6 \%)$ & $263(53.1 \%)$ & $0.80(0.62-1.04)$ & & $0.88(0.59-1.34)$ & \\
\hline & Log-additive & - & - & - & $1.03(0.85-1.24)$ & 0.780 & $0.84(0.62-1.14)$ & 0.260 \\
\hline
\end{tabular}


Table 4 (continued)

\begin{tabular}{|c|c|c|c|c|c|c|c|c|}
\hline \multirow[t]{2}{*}{ SNP-ID } & \multirow[t]{2}{*}{ Model } & \multirow[t]{2}{*}{ Genotype } & \multirow[t]{2}{*}{ Case, \% } & \multirow[t]{2}{*}{ Control, \% } & \multicolumn{2}{|c|}{ Without adjustment } & \multicolumn{2}{|l|}{ With adjustment } \\
\hline & & & & & OR $(95 \% \mathrm{CI})$ & $p$ & OR $(95 \%$ CI $)$ & $p$ \\
\hline \multirow[t]{10}{*}{ rs11896604 } & Codominant & $\mathrm{C} / \mathrm{C}$ & $259(62.3 \%)$ & 317 (64\%) & 1.00 & 0.220 & 1.00 & 0.310 \\
\hline & & $\mathrm{C} / \mathrm{G}$ & $136(32.7 \%)$ & $164(33.1 \%)$ & $1.01(0.77-1.34)$ & & $0.82(0.53-1.29)$ & \\
\hline & & $\mathrm{G} / \mathrm{G}$ & $21(5 \%)$ & $14(2.8 \%)$ & $1.84(0.92-3.68)$ & & $1.98(0.63-6.26)$ & \\
\hline & Dominant & $\mathrm{C} / \mathrm{C}$ & $259(62.3 \%)$ & 317 (64\%) & 1.00 & 0.580 & 1.00 & 0.600 \\
\hline & & $\mathrm{C} / \mathrm{G}-\mathrm{G} / \mathrm{G}$ & $157(37.7 \%)$ & $178(36 \%)$ & $1.08(0.82-1.41)$ & & $0.89(0.58-1.37)$ & \\
\hline & Recessive & $\mathrm{C} / \mathrm{C}-\mathrm{C} / \mathrm{G}$ & 395 (95\%) & $481(97.2 \%)$ & 1.00 & 0.083 & 1.00 & 0.200 \\
\hline & & $\mathrm{G} / \mathrm{G}$ & $21(5 \%)$ & $14(2.8 \%)$ & $1.83(0.92-3.64)$ & & $2.11(0.67-6.60)$ & \\
\hline & Overdominant & $\mathrm{C} / \mathrm{C}-\mathrm{G} / \mathrm{G}$ & $280(67.3 \%)$ & $331(66.9 \%)$ & 1.00 & 0.890 & 1.00 & 0.310 \\
\hline & & $\mathrm{C} / \mathrm{G}$ & $136(32.7 \%)$ & $164(33.1 \%)$ & $0.98(0.74-1.29)$ & & $0.80(0.51-1.24)$ & \\
\hline & Log-additive & - & - & - & $1.13(0.90-1.43)$ & 0.290 & $0.99(0.68-1.44)$ & 0.970 \\
\hline \multirow[t]{10}{*}{ rs17045754 } & Codominant & $\mathrm{G} / \mathrm{G}$ & $270(64.9 \%)$ & $325(65.7 \%)$ & 1.00 & 0.370 & 1.00 & 0.590 \\
\hline & & $\mathrm{G} / \mathrm{C}$ & $128(30.8 \%)$ & $157(31.7 \%)$ & $0.98(0.74-1.30)$ & & $0.86(0.55-1.35)$ & \\
\hline & & $\mathrm{C} / \mathrm{C}$ & $18(4.3 \%)$ & $13(2.6 \%)$ & $1.67(0.80-3.46)$ & & $1.54(0.46-5.15)$ & \\
\hline & Dominant & $\mathrm{G} / \mathrm{G}$ & $270(64.9 \%)$ & $325(65.7 \%)$ & 1.00 & 0.810 & 1.00 & 0.660 \\
\hline & & $\mathrm{G} / \mathrm{C}-\mathrm{C} / \mathrm{C}$ & $146(35.1 \%)$ & $170(34.3 \%)$ & $1.03(0.79-1.36)$ & & $0.91(0.59-1.40)$ & \\
\hline & Recessive & $\mathrm{G} / \mathrm{G}-\mathrm{G} / \mathrm{C}$ & $398(95.7 \%)$ & $482(97.4 \%)$ & 1.00 & 0.160 & 1.00 & 0.430 \\
\hline & & $\mathrm{C} / \mathrm{C}$ & $18(4.3 \%)$ & $13(2.6 \%)$ & $1.68(0.81-3.46)$ & & $1.62(0.49-5.35)$ & \\
\hline & Overdominant & $\mathrm{G} / \mathrm{G}-\mathrm{C} / \mathrm{C}$ & $288(69.2 \%)$ & $338(68.3 \%)$ & 1.00 & 0.760 & 1.00 & 0.450 \\
\hline & & $\mathrm{G} / \mathrm{C}$ & $128(30.8 \%)$ & $157(31.7 \%)$ & $0.96(0.72-1.27)$ & & $0.84(0.54-1.32)$ & \\
\hline & Log-additive & - & - & - & $1.08(0.86-1.37)$ & 0.500 & $0.97(0.66-1.42)$ & 0.890 \\
\hline \multirow[t]{10}{*}{ rs843720 } & Codominant & $\mathrm{T} / \mathrm{T}$ & $154(37)$ & $213(43)$ & 1.00 & 0.100 & 1.00 & 0.083 \\
\hline & & $\mathrm{G} / \mathrm{T}$ & $204(49)$ & $230(46.5)$ & $1.23(0.93-1.62)$ & & $1.45(0.93-2.27)$ & \\
\hline & & $\mathrm{G} / \mathrm{G}$ & 58 (13.9) & $52(10.5)$ & $1.54(1.01-2.37)$ & & $2.01(1.02-3.96)$ & \\
\hline & Dominant & $\mathrm{T} / \mathrm{T}$ & $154(37)$ & $213(43)$ & 1.00 & 0.065 & 1.00 & 0.044 \\
\hline & & $\mathrm{G} / \mathrm{T}-\mathrm{G} / \mathrm{G}$ & $262(63)$ & $282(57)$ & $1.29(0.98-1.68)$ & & $1.55(1.01-2.37)$ & \\
\hline & Recessive & $\mathrm{T} / \mathrm{T}-\mathrm{G} / \mathrm{T}$ & $358(86.1)$ & $443(89.5)$ & 1.00 & 0.110 & 1.00 & 0.130 \\
\hline & & $\mathrm{G} / \mathrm{G}$ & 58 (13.9) & $52(10.5)$ & $1.38(0.93-2.06)$ & & $1.63(0.87-3.06)$ & \\
\hline & Overdominant & $\mathrm{T} / \mathrm{T}-\mathrm{G} / \mathrm{G}$ & $212(51)$ & $265(53.5)$ & 1.00 & 0.440 & 1.00 & 0.330 \\
\hline & & $\mathrm{G} / \mathrm{T}$ & $204(49)$ & $230(46.5)$ & $1.11(0.85-1.44)$ & & $1.23(0.81-1.86)$ & \\
\hline & Log-additive & - & - & - & $1.24(1.02-1.51)$ & 0.033 & $1.43(1.04-1.95)$ & 0.026 \\
\hline
\end{tabular}

SNP, single nucleotide polymorphism; OR, odds ratio; 95\% CI, 95\% confidence interval. $p$ value $<0.05$ indicates statistical significance. $p$ value $<0.004(0.05 / 11)$ indicates statistical significance after Bonferroni correction.

$\mathrm{OR}=3.33,95 \% \mathrm{CI}: 1.05-10.51, p=0.042 ; \mathrm{OR}=2.22,95 \% \mathrm{CI}: 1.05-4.68, p=0.032$ ) in the recessive model. In addition, compared with the TT genotype, the GT and GG genotypes of rs843720 were associated with 1.55 -fold increased IgAN risk in the dominant model (adjusted OR $=1.55,95 \%$ CI: $1.01-2.37, p=0.044$ ). And rs843720 was significantly associated with an increased risk of IgAN in the log-additive model (adjusted OR $=1.43,95 \%$ CI: 1.04-1.95, $p=$ 0.026; $\mathrm{OR}=1.24,95 \% \mathrm{CI}: 1.02-1.51, p=0.033$ ) (Table 4). After Bonferroni correction, the significance of multiplex detection at the $p<0.004(0.05 / 11)$ level was not retained for rs12615793 and rs843720. In addition, no association was found between other polymorphisms of ACYP2 and IgAN risk in the genetic models. 
Jin et al.: ACYP2 Polymorphisms and IgAN Risk

Table 5. Association between haplotypes of ACYP2 and IgAN risk

\begin{tabular}{|c|c|c|c|c|c|c|c|c|c|}
\hline \multirow[t]{2}{*}{ SNP-ID } & \multirow[t]{2}{*}{ Haplotype } & \multirow[t]{2}{*}{ F_A } & \multirow[t]{2}{*}{$\mathrm{F}_{-} \mathrm{U}$} & \multirow[t]{2}{*}{$\chi^{2}$} & \multirow[t]{2}{*}{$p^{\mathrm{a}}$} & \multicolumn{2}{|c|}{ Without adjustment } & \multicolumn{2}{|l|}{ With adjustment } \\
\hline & & & & & & OR $(95 \% \mathrm{CI})$ & $p^{\mathrm{b}}$ & OR $(95 \% \mathrm{CI})$ & $p^{\mathrm{b}}$ \\
\hline \multirow{5}{*}{$\begin{array}{l}\text { rs1682111/rs843752/ } \\
\text { rs843645/rs11125529/ } \\
\text { rs12615793/rs843711/ } \\
\text { rs11896604 }\end{array}$} & ATTCGCC & 0.323 & 0.303 & 0.886 & 0.346 & 1.00 & - & 1.00 & - \\
\hline & TGGCGTC & 0.239 & 0.257 & 0.778 & 0.378 & $0.87(0.67-1.12)$ & 0.280 & $0.75(0.51-1.12)$ & 0.160 \\
\hline & TTTCGCC & 0.195 & 0.225 & 2.397 & 0.122 & $0.80(0.62-1.05)$ & 0.110 & $0.89(0.58-1.36)$ & 0.580 \\
\hline & TTTAATG & 0.194 & 0.179 & 0.696 & 0.404 & $1.02(0.77-1.34)$ & 0.900 & $0.88(0.56-1.37)$ & 0.570 \\
\hline & TTTCACC & 0.014 & 0.011 & 0.406 & 0.524 & $1.16(0.49-2.74)$ & 0.730 & $1.10(0.30-4.03)$ & 0.890 \\
\hline
\end{tabular}

SNP, single nucleotide polymorphism; F_A, frequency in cases; F_U, frequency in controls; OR, odds ratio; 95\% CI, 95\% confidence interval. ${ }^{a} p$ values were calculated using the Pearson's $\chi^{2}$ test. ${ }^{\mathrm{b}} p$ values were calculated using the Wald test. $p<0.05$ indicates statistical significance.

\section{Haplotype Analysis}

Seven SNPs in the ACYP2, including rs1682111, rs843752, rs843645, rs11125529, rs12615793, rs843711, and rs11896604 showed significant LD, as shown in Figure 1. Five haplotypes were revealed after the combination of seven SNPs in ACYP2. When the putative risk genotypes were combined and used as the reference ATTCGCC haplotype, no significant difference in the haplotype frequency distributions between the IgAN patient and control groups was found $(p>0.05)$. The results are presented in Table 5.

\section{SNP Genotypes and Clinical Indicator Levels}

We compared the mean levels of clinical indicators between three genotypes of rs843720 (Table 6). There was significant difference in the mean level of HB between the GT genotype and TT genotype groups with IgAN $(p<0.05)$. However, no significant difference in the mean levels of other clinical indicators was found between the GT genotype and TT genotype groups, and the GG genotype and TT genotype groups with IgAN $(p>0.05)$.

\section{Discussion}

To date, there have been no reports on the correlation between the polymorphisms of $A C Y P 2$ and IgAN risk. In this case-control study, we aimed to investigate the association between polymorphisms of ACYP2 and the risk of IgAN in the Chinese Han population. The results revealed that the rs $843720 \mathrm{G}$ allele significantly increased the risk of IgAN, and rs843720 was associated with increased risk in both the dominant model and log-additive model before Bonferroni correction. In addition, we found that rs12615793 was also significantly associated with increased risk of IgAN in the recessive model. However, no significant difference in the distribution of allele and genotype was observed in other nine SNPs of ACYP2 between the IgAN patients and the controls $(p>0.05)$.

ACYP2 (2p16.2) encodes a cytosolic enzyme acylphosphatase, which can adjust the stability of the inner environment of $\mathrm{Ca}^{2+}[15]$, and plays a role in pyruvate metabolism [28], cell differentiation [29], senescence [30], and apoptosis [31]. Recently, a genome-wide metaanalysis had successfully identified that rs11125529 was associated with shorter telomere length in the European population [17]. In humans, telomere shortening takes place in many tissues with age, and is accelerated in chronic diseases and cancer [32]. Some reports have identified that telomere shortening is associated with the progression of IgAN $[18,19]$. However, we did not observe any significant association between rs11125529 and the risk of IgAN. One possibility is that rs11125529 may not correlate with shorter telomere length in 


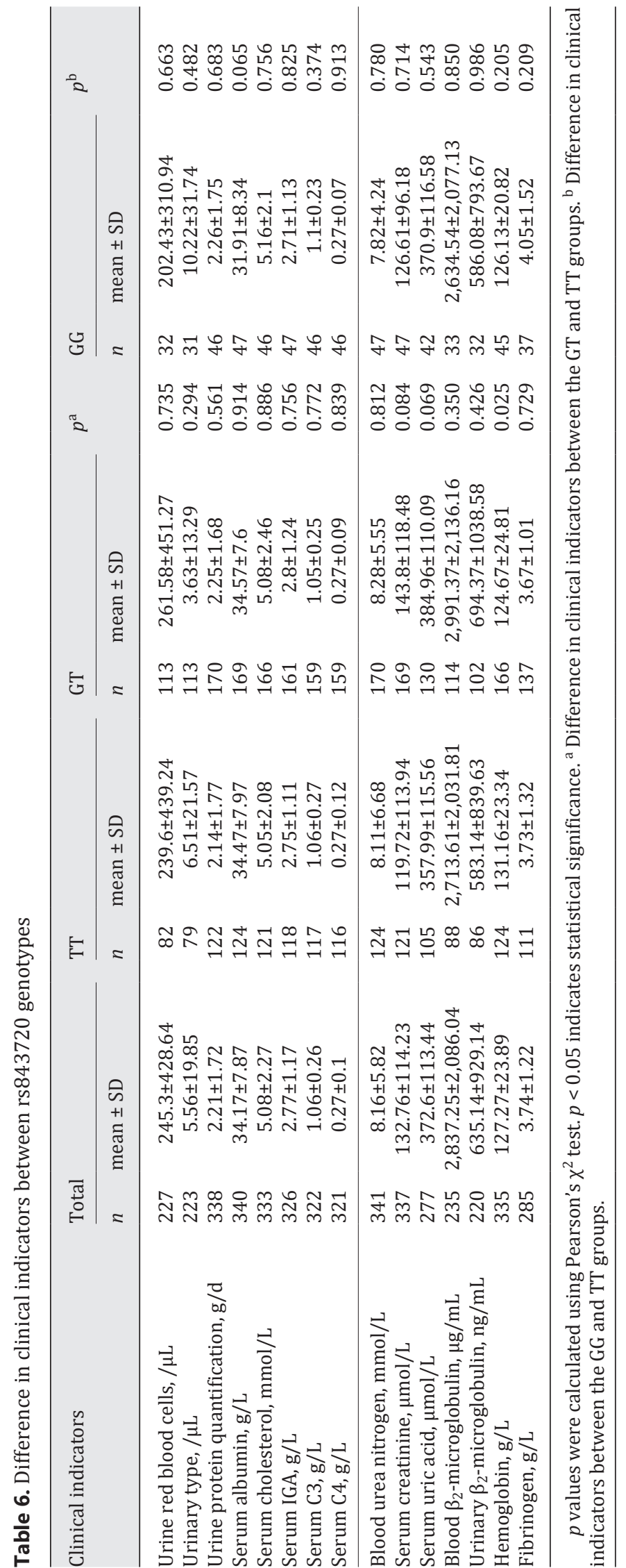




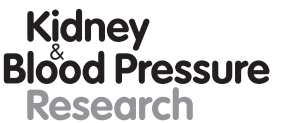

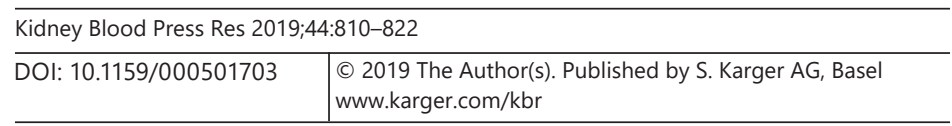

Jin et al.: ACYP2 Polymorphisms and IgAN Risk

the Chinese Han population. There is another possibility that rs11125529 is a consequence of the IgAN rather than occurring prior to disease development. It will be necessary to reveal the detailed causes in further studies.

The results of this study demonstrated that rs843720 and rs12615793 significantly increased the risk of IgAN. Therefore, the association provided evidence that rs843720 and rs12615793 might be influential factors for telomere length and increase the risk of IgAN by affecting telomere length. However, no difference in the mean levels of clinical indicators (except for HB) was found between the wild-type and mutant types of the rs843720 groups. The finding suggested that rs843720 may increase the risk of IgAN through other mechanisms. Moreover, the $\mathrm{Ca}^{2+} / \mathrm{Mg}^{2+}$-ATPase activity was found to be reduced, and $\mathrm{Ca}^{2+}$ homeostasis is disturbed in children with chronic kidney disease [16]. It may be that rs843720 and rs12615793 affect the $\mathrm{Ca}^{2+} / \mathrm{Mg}^{2+}$-ATPase activity and calcium homeostasis and thus affect the development of kidney disease [16]. Therefore, further studies are required to investigate the detailed mechanisms of IgAN development.

The rs 843720 has been reported to be significantly correlated with increased lung cancer risk in the recessive model [22]. Moreover, previous research showed that the AA genotype of rs12615793 was associated with a decreased risk of high-altitude pulmonary edema, compared to the GG and AG genotypes [33], but allele A of rs12615793 was found to be associated with an increased risk of ischemic stroke in the Chinese Han population [24]. This suggests that polymorphisms may affect diseases differently.

This study has some limitations. First, the study sample is relatively small, which limits the ability to determine the association between ACYP2 polymorphisms and the risk of IgAN. Second, a more closely matched population may reveal differences that were not found in this study, as the characteristics of the healthy control group were significantly different from those of the IgAN patients group. Third, many other risk factors (e.g., smoking, drinking) and clinical and pathological characteristics were not included. Finally, the biological function analyses of $A C Y P 2$ polymorphisms were not performed.

\section{Conclusions}

In conclusion, this study indicated that rs843720 and rs12615793 are associated with increased risk of IgAN in the Chinese Han population, which suggested the two polymorphisms might play a role in the development of IgAN in the Chinese Han population. However, further analysis must be performed with a larger sample size to enhance the power of the study and expand the knowledge of the functions of ACYP2 polymorphisms in the development of IgAN.

\section{Acknowledgments}

The study was supported by the Shaanxi Science and Technology Research and Development Project Fund (No. S2016YFSF0644). We are grateful to the patients and control individuals for their participation in the study. We also thank the clinicians and hospital staff who contributed to sample and data collection for this study. 


\section{Kidney \\ Blood Pressure \\ Research}

\begin{tabular}{l|l}
\hline Kidney Blood Press Res 2019;44:810-822 \\
\hline DOI: 10.1159/000501703 & $\begin{array}{l}\text { @ 2019 The Author(s). Published by S. Karger AG, Basel } \\
\text { www.karger.com/kbr }\end{array}$ \\
\hline
\end{tabular}

Jin et al.: ACYP2 Polymorphisms and IgAN Risk

\section{Statement of Ethics}

All subjects provided written informed consent to voluntarily participate in this study. This study was approved by the Medical Ethics Committee of the First Affiliated Hospital of Xi'an Jiaotong University.

\section{Disclosure Statement}

The authors have declared that no competing interest exists.

\section{References}

1 Wyatt RJ, Julian BA. IgA nephropathy. N Engl J Med. 2013 Jun;368(25):2402-14.

2 Schena FP. A retrospective analysis of the natural history of primary IgA nephropathy worldwide. Am J Med. 1990 Aug;89(2):209-15.

3 D'Amico G. Natural history of idiopathic IgA nephropathy: role of clinical and histological prognostic factors. Am J Kidney Dis. 2000 Aug;36(2):227-37.

4 Lv J, Zhang H, Zhou Y, Li G, Zou W, Wang H. Natural history of immunoglobulin A nephropathy and predictive factors of prognosis: a long-term follow up of 204 cases in China. Nephrology (Carlton). 2008 Jun;13(3):242-6.

5 Wakai K, Kawamura T, Matsuo S, Hotta N, Ohno Y. Risk factors for IgA nephropathy: a case-control study in Japan. Am J Kidney Dis. 1999 Apr;33(4):738-45.

6 Hsu SI, Ramirez SB, Winn MP, Bonventre JV, Owen WF. Evidence for genetic factors in the development and progression of IgA nephropathy. Kidney Int. 2000 May;57(5):1818-35.

7 Kiryluk K, Li Y, Sanna-Cherchi S, Rohanizadegan M, Suzuki H, Eitner F, et al. Geographic differences in genetic susceptibility to IgA nephropathy: GWAS replication study and geospatial risk analysis. PLoS Genet. 2012; 8(6):e1002765.

8 Yu XQ, Li M, Zhang H, Low HQ, Wei X, Wang JQ, et al. A genome-wide association study in Han Chinese identifies multiple susceptibility loci for IgA nephropathy. Nat Genet. 2011 Dec;44(2):178-82.

9 Bisceglia L, Cerullo G, Forabosco P, Torres DD, Scolari F, Di Perna M, et al.; European IgAN Consortium. Genetic heterogeneity in Italian families with IgA nephropathy: suggestive linkage for two novel IgA nephropathy loci. Am J Hum Genet. 2006 Dec;79(6):1130-4.

10 Niu D, Ren Y, Xie L, Sun J, Lu W, Hao Y, et al. Association between CCDC132, FDX1 and TNFSF13 gene polymorphisms and the risk of IgA nephropathy. Nephrology (Carlton). 2015 Dec;20(12):908-15.

11 Gao J, Wei L, Liu X, Wang L, Niu D, Jin T, et al. Association Between IFN- $\gamma$ Gene Polymorphisms and IgA Nephropathy in a Chinese Han Population. Kidney Blood Press Res. 2017;42(1):136-44.

12 Gao J, Liu X, Wei L, Niu D, Wei J, Wang L, et al. Genetic variants of MCP-1 and CCR2 genes and IgA nephropathy risk. Oncotarget. 2016 Nov;7(47):77950-7.

13 Gao J, Wei L, Wei J, Yao G, Wang L, Wang M, et al. TLR1 polymorphism rs4833095 as a risk factor for IgA nephropathy in a Chinese Han population: A case-control study. Oncotarget. 2016 Dec;7(50):83031-9.

14 Modesti A, Raugei G, Taddei N, Marzocchini R, Vecchi M, Camici G, et al. Chemical synthesis and expression of a gene coding for human muscle acylphosphatase. Biochim Biophys Acta. 1993 Dec;1216(3):369-74.

15 Degl'Innocenti D, Marzocchini R, Rosati F, Cellini E, Raugei G, Ramponi G. Acylphosphatase expression during macrophage differentiation and activation of U-937 cell line. Biochimie. 1999 Nov;81(11):1031-5.

16 Polak-Jonkisz D, Purzyc L, Laszki-Szczachor K, Musial K, Zwolinska D. The endogenous modulators of Ca2+Mg2+-dependent ATPase in children with chronic kidney disease (CKD). Nephrol Dial Transplant. 2010 Feb; 25(2):438-44.

17 Codd V, Nelson CP, Albrecht E, Mangino M, Deelen J, Buxton JL, et al.; CARDIoGRAM consortium. Identification of seven loci affecting mean telomere length and their association with disease. Nat Genet. 2013 Apr; 45(4): 422-7.

18 Lu YY, Yang X, Chen WQ, Ju ZY, Shou ZF, Jin J, et al. Proteins induced by telomere dysfunction are associated with human IgA nephropathy. J Zhejiang Univ Sci B. 2014 Jun;15(6):566-74.

19 Szeto CC, Poon PY, Lai FM, Chow KM, Szeto CY, Li PK. Chromosomal telomere shortening of kidney cells in IgA nephropathy by the measurement of DNA in urinary sediment. Clin Nephrol. 2005 Nov;64(5):337-42.

20 Zhu L, Liu L, He X, Yan M, Du J, Yang H, et al. Association between genetic polymorphism of telomere-associated gene ACYP2 and the risk of HAPE among the Chinese Han population: A case-control study. Medicine (Baltimore). 2017 Mar;96(13):e6504.

21 Zhang F, Zhang Y, Deng Z, Xu P, Zhang X, Jin T, et al. Genetic variants in the acylphosphatase 2 gene and the risk of breast cancer in a Han Chinese population. Oncotarget. 2016 Dec;7(52):86704-12. 
22 Chen N, Yang X, Guo W, You J, Wu Q, Zhang G, et al. Association of polymorphisms in the telomere-related gene ACYP2 with lung cancer risk in the Chinese Han population. Oncotarget. 2016 Dec;7(52):87473-8.

$23 \mathrm{Li} \mathrm{J}$, Ma G, Zhu X, Jin T, Wang J, Li C. Association analysis of telomere length related gene ACYP2 with the gastric cancer risk in the northwest Chinese Han population. Oncotarget. 2017 May;8(19):31144-52.

24 Liang Y, Zhang R, Zhang S, Ji G, Shi P, Yang T, et al. Association of ACYP2 and TSPYL6 Genetic Polymorphisms with Risk of Ischemic Stroke in Han Chinese Population. Mol Neurobiol. 2017 Oct;54(8):5988-5995.

25 Huang CY, Xun XJ, Wang AJ, Gao Y, Ma JY, Chen YT, et al. CHRNA5 polymorphisms and risk of lung cancer in Chinese Han smokers. Am J Cancer Res. 2015 Sep;5(10):3241-8.

26 Hu Q, Zhang M, He N, Liang G, Xu S, Wang Y, Mo F: Association of genetic polymorphisms with pulmonary tuberculosis in a Chinese Tibetan population: a case-control study.

27 Geng TT, Xun XJ, Li S, Feng T, Wang LP, Jin TB, et al. Association of colorectal cancer susceptibility variants with esophageal cancer in a Chinese population. World J Gastroenterol. 2015 Jun;21(22):6898-904.

28 Yu WS, Jeong SJ, Kim JH, Lee HJ, Song HS, Kim MS, et al. The genome-wide expression profile of 1,2,3,4,6-penta0-galloyl- $\beta$-D-glucose-treated MDA-MB-231 breast cancer cells: molecular target on cancer metabolism. Mol Cells. 2011 Aug;32(2):123-32.

29 Chiarugi P, Degl'Innocenti D, Taddei L, Raugei G, Berti A, Rigacci S, et al. Acylphosphatase is involved in differentiation of K562 cells. Cell Death Differ. 1997 May;4(4):334-40.

30 Fiaschi T, Chiarugi P, Veggi D, Raugei G, Ramponi G. The inhibitory effect of the $5^{\prime}$ untranslated region of muscle acylphosphatase mRNA on protein expression is relieved during cell differentiation. FEBS Lett. 2000 May;473(1):42-6.

31 Giannoni E, Cirri P, Paoli P, Fiaschi T, Camici G, Manao G, et al. Acylphosphatase is a strong apoptosis inducer in HeLa cell line. Mol Cell Biol Res Commun. 2000 May;3(5):264-70.

32 Jiang H, Ju Z, Rudolph KL. Telomere shortening and ageing. Z Gerontol Geriatr. 2007 Oct;40(5):314-24.

33 He Y, Zhang X, Li X, Du J, He X, Zhang Z, et al. Telomere length-related gene ACYP2 polymorphism is associated with the risk of HAPE in Chinese Han population. J Gene Med. 2016 Sep;18(9):244-9. 